\title{
The origins of interneuron diversity
}

DOI:

10.1038/nrn2225

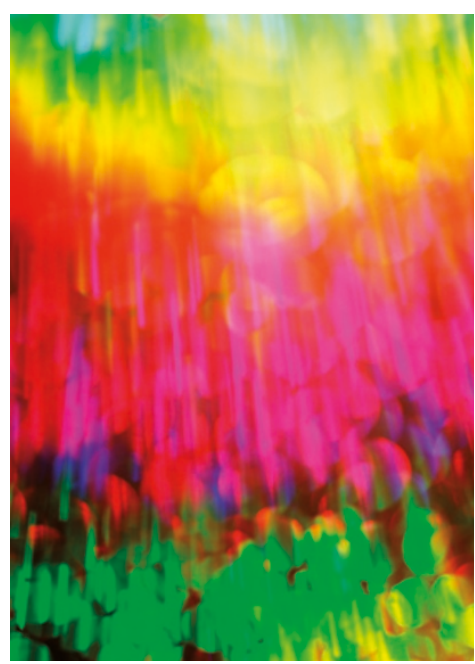

The enormous diversity of cortical interneuron subtypes presents a challenge for our understanding of their functional roles. A study by Fishell and colleagues examines the origins of interneuron diversity during development, highlighting the importance of an interneuron's birth order in establishing its physiological properties.

Cortical interneurons arise from the medial and caudal ganglionic eminences (MGE and CGE, respectively) in the ventral telencephalic ventricular zone. The order in which they are born correlates with their eventual laminar position within the cortex. However, it is not known how the generation of multiple subtypes of interneuron - each with different physiological, morphological and molecular properties - is coordinated.

To examine whether temporal birth order relates to interneuron subtype, the authors carried out mosaic fate mapping. They used transgenic mice in which the expression of a fluorescent protein can be switched on by the administration of tamoxifen in cells that are expressing Olig2, a gene that is highly expressed in MGE progenitors. Using this strategy, they labelled neural precursors at embryonic days (E) 9.5, 10.5, 12.5 and 15.5 and then examined the properties of the progeny that were born at these time points.

The authors first examined the molecular expression profiles of labelled interneurons in the somatosensory barrel field at 21 days after birth. They defined seven different molecular profiles, based on the expression of combinations of the interneuron markers parvalbumin (PV), somatostatin (SST), vasoactive intestinal polypeptide (VIP) and calretinin (CR). This revealed that, whereas PV-expressing interneurons were generated continuously between E9.5 and E15.5, SST-positive and CR-negative interneurons were principally born at early developmental stages. By contrast, interneurons that expressed either CR or VIP were born primarily at E15.5.

The electrophysiological properties of interneurons are often used to classify specific subtypes. Using 26 physiological parameters, and adhering broadly to the classification system that arose from a 2005 meeting in Petilla, the authors categorized 205 interneurons into ten subtypes and looked for correlations between the subtype and the neurons' birth order. This revealed some broad patterns: most 'fast-spiking' interneurons were born between E9.5 and E12.5; however, a set of 'delayed fast-spiking' interneurons arose largely from cells that were labelled at E15.5. Most 'intrinsic bursting' neurons were born between E9.5 and E10, and two groups of 'non-fast-spiking' interneurons (NFS1 and NFS2) were born at approximately E12.5. The 'delayed non-fast-spiking type 2', 'late-spiking' and 'initial-adapting' subtypes were all mostly born at E15.5. Finally, the 'delayed non-fastspiking type 1' subtype showed a bimodal birth pattern, with peaks at E9.5 and E15.5.

This study suggests that an interneuron's birth date may significantly affect its physiological and molecular properties. Although much remains to be learned about the populations from which cells that are born at different time points are derived and how switches in cell fate might be made, this work suggests that developmental genetics has much to contribute to the study of interneuron diversity and classification.

Katherine Whalley

ORIGINAL RESEARCH PAPER Miyoshi, G.

Butt, S. J., Takebayashi, H. \& Fishell, G.

Physiologically distinct temporal cohorts of cortical interneurons arise from telencephalic Olig2-expressing precursors. J. Neurosci. 27, 7786-7798 (2007)

FURTHER READING Wonders, C.P. \& Anderson, S. A. The origin and specification of cortical interneurons. Nature Rev. Neurosci. 7 , 687-696 (2006)| Huang, Z. J., Di Cristo, G. \& Ango F. Development of GABA innervation in cerebral and cerebellar cortex. Nature Rev. Neurosci. 8, 673-686 (2007) 